\title{
Signature of gate-controlled magnetism and localization effects at $\mathrm{Bi}_{2} \mathrm{Se}_{3} / \mathrm{EuS}$ interface
}

\author{
Subramanian Mathimalar ${ }^{1,5}$, Satyaki Sasmal ${ }^{1,5}$, Archit Bhardwaj ${ }^{1}$, Sekar Abhaya ${ }^{2,3}$, Rajasekhar Pothala ${ }^{1}$, Saurabh Chaudhary ${ }^{1}$, \\ Biswarup Satpati ${ }^{4}$ and Karthik V. Raman iD $^{1 凶}$
}

Proximity of a topological insulator ( $\mathrm{TI})$ surface with a magnetic insulator (MI) can open an exchange gap at the Dirac point leading to exploration of surface quantum anomalous Hall effect. An important requirement to observe the above effect is to prevent the topological breakdown of the surface states (SSs) due to various interface coupling effects and to tune the Fermi level at the interface near the Dirac point. In this work, we demonstrate the growth of high-quality c-axis oriented strain-free layered films of $\mathrm{Tl}_{1}, \mathrm{Bi}_{2} \mathrm{Se}_{3}$, on amorphous $\mathrm{SiO}_{2}$ substrate in proximity to an $\mathrm{Ml}$, europium sulfide (EuS), that show stronger weak anti-localization response from the surface than previous studies with epitaxially interfaced heterostructures. Importantly, we find gate and magnetic field cooling modulated localization effects in the SSs, attributed to the position of interface Fermi level within the band gap that is also corroborated from our positron annihilation spectroscopy measurements. Furthermore, our experiments provide a direct evidence of gate-controlled enhanced interface magnetism in EuS arising from the carrier mediated Ruderman-Kittel-Kasuya-Yosida interactions across the $\mathrm{Bi}_{2} \mathrm{Se}_{3} /$ EuS interface. These findings demonstrate the existence of complex interfacial phenomena affecting the localization response of the SSs that might be important in proximity engineering of the TI surface to observe surface quantum Hall effects.

npj Quantum Materials (2020)5:64; https://doi.org/10.1038/s41535-020-00267-5

\section{INTRODUCTION}

The process of breaking time reversal symmetry by introducing out-of-plane magnetism in the topologically protected surface states (SSs) of a 3-dimensional (3D) topological insulator (TI) ${ }^{1,2}$ leads to opening of an exchange gap (EG) at the Dirac point ${ }^{3}$. Such a mechanism support several new quantum phenomena, such as quantum anomalous Hall effect (QAHE) ${ }^{4,5}$, half-integer quantum Hall effect $(\mathrm{H}-\mathrm{IQHE})^{1,6}$, topological magneto-electric effect ${ }^{7,8}$, and image magnetic monopoles ${ }^{9}$. In realizing these effects, the main challenge is to successfully demonstrate the presence of the EG with the Fermi level $\left(E_{F}\right)$ lying within the gap. Currently, this is made possible in magnetic Tls such as in $\mathrm{Cr}$ - and V-doped compensated Tls, where perpendicular magnetism is developed in the bulk showing $Q A H E^{5,10}$. However, in comparison, inducing local magnetism at $\mathrm{TI}$ surface through the short range nature of magnetic proximity effect (MPE) can provide a number of advantages over bulk doping ${ }^{3,5,11}$; these include lower bulk and surface defect density due to improved film crystallinity, better controllability of the SSs, and independently controlling the magnetic state at each surface of $\mathrm{TI}$ to build switchable topological devices ${ }^{12}$.

Conventionally, MPE can be achieved by using magnetic insulators (MIs). However, requirement of an out-of-plane magnetization at the TI surface makes this study difficult since most Mls naturally possess in-plane anisotropy. Also, new hybridization states $^{13}$ that may form at the TI/MI interface near the $E_{F}$ can contaminate the topological properties of the Dirac SSs ${ }^{14-16}$. As a result, observing these effects through transport studies can become nontrivial.

In this regard, MPE studies in the 3D TI, Bismuth Selenide $\left(\mathrm{Bi}_{2} \mathrm{Se}_{3}\right)$, with an $\mathrm{Ml}$, europium sulfide (EuS), is extensively researched ${ }^{17-19}$. Different research groups confirm the presence of an out-of-plane component or canted magnetization at the $\mathrm{Bi}_{2} \mathrm{Se}_{3} /$ EuS interface with the magnetism existing at temperature much above the magnetic transition temperature $\left(T_{c}\right)$ of EuS $(\sim 17 \mathrm{~K})^{18,20}$. Our magnetometry studies on $\mathrm{Bi}_{2} \mathrm{Se}_{3}(10 \mathrm{~nm}) / \mathrm{EuS}$ $(1 \mathrm{~nm})$ also confirm the existence of enhanced interface magnetism with a second transition close to $232 \mathrm{~K}$ (refer to Supplementary Fig. 2 and Supplementary Note 2). Unlike other TI/MI interfaces $^{14,15,21,22}$, recent theoretical calculation of the above interface also suggests induced magnetism, localized within the first quintuple layer $(\mathrm{QL})$ of $\mathrm{Bi}_{2} \mathrm{Se}_{3}{ }^{23}$, with the $\mathrm{SS}$ retaining most of the topological character compared to a pristine $\mathrm{Bi}_{2} \mathrm{Se}_{3} \mathrm{SSs}^{20}$. Furthermore, an induced EG of $\sim 9 \mathrm{meV}$ in the surface Dirac cone is suggested ${ }^{23}$. Despite these reports, experimental observation of the EG opening at this surface has been elusive ${ }^{24}$. Possible reasons for the above include: (a) inherent intrinsic doping from large Se vacancies leading to bulk dominated conduction with $E_{F}$ pinning inside the conduction band (CB), and (b) loss of topological character/signal due to interface hybridization, surface-bulk coupling effects ${ }^{25}$, and/or due to reduction in thickness (in an effort to probe the surface contribution over the bulk conduction) that weaken the robustness of bulk bands to preserve the SSs.

In literature, the topological nature of SSs and its response to MPE are probed using magnetoconductance measurements by analyzing the weak anti-localization (WAL) or weak-localization (WL) signal from the spin-momentum locked $\mathrm{SS}$. In $\mathrm{Bi}_{2} \mathrm{Se}_{3}$, this signal is weak due to the dominant bulk contribution to conductivity, thereby making them harder to detect electronically. Reducing bulk conduction in $\mathrm{Bi}_{2} \mathrm{Se}_{3}$ films is achieved either by studying thinner films, or by reducing the bulk conductivity. Previous studies with epitaxially interfaced $\mathrm{Bi}_{2} \mathrm{Se}_{3} /$ EuS films ${ }^{17}$,

\footnotetext{
${ }^{1}$ Tata Institute of Fundamental Research, Hyderabad 500107, India. ${ }^{2}$ Materials Science Group, Indira Gandhi Centre for Atomic Research, Kalpakkam, Tamil Nadu 603102, India. ${ }^{3}$ Homi Bhabha National Institute, Kalpakkam, Tamil Nadu 603102, India. ${ }^{4}$ Saha Institute of Nuclear Physics, 1/AF Bidhannagar, Kolkata 700064, India. ${ }^{5}$ These authors contributed equally: Subramanian Mathimalar, Satyaki Sasmal. ${ }^{凶}$ email: kvraman@tifrh.res.in
} 

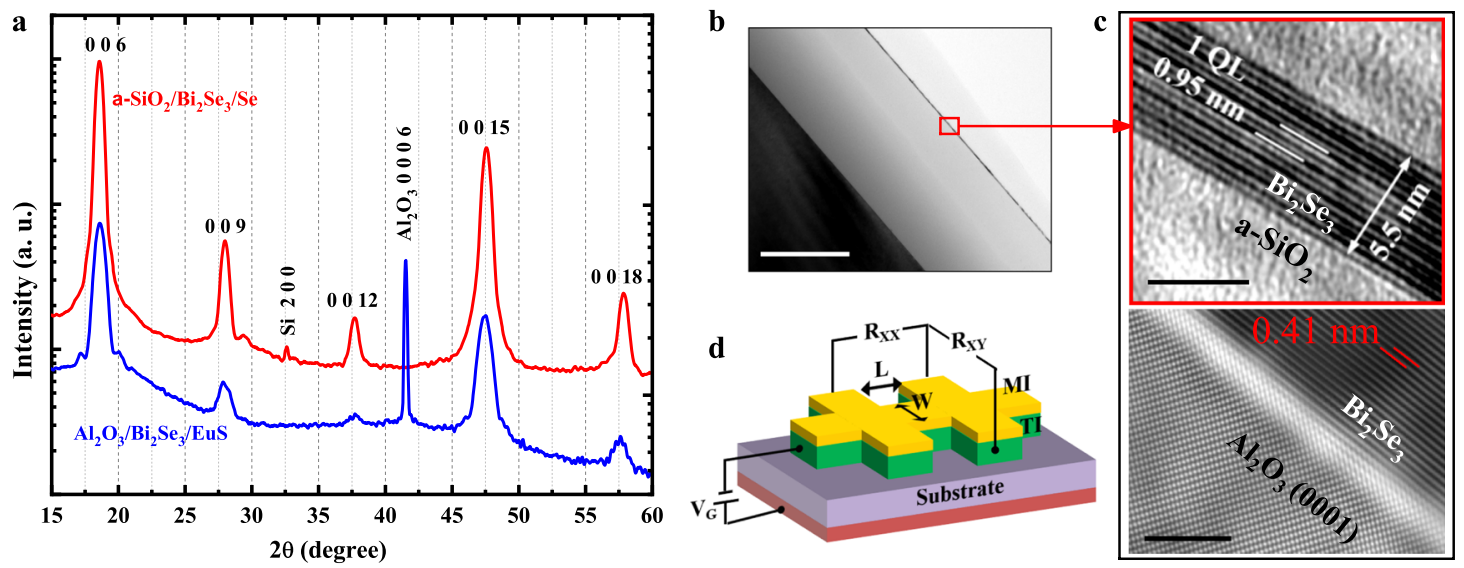

Fig. 1 XRD, TEM, and device structure. a XRD pattern in $\theta-2 \theta$ scan of $\mathrm{Bi}_{2} \mathrm{Se}_{3}$ films grown on sapphire (0001) and a-SiO ${ }_{2}$ substrate. $\mathbf{b}$ Crosssectional TEM image (zoomed out view) of $\mathrm{Bi}_{2} \mathrm{Se}_{3}$ films grown on a-SiO ${ }_{2}$. Length of the scale bar is $200 \mathrm{~nm}$. c Zoom-in-view of cross-sectional TEM image of $\mathrm{Bi}_{2} \mathrm{Se}_{3}$ on a-SiO $\mathrm{Stop}_{2}$ and sapphire (0001) (bottom). Length of the scale bars is $5 \mathrm{~nm}$. d Schematic of our Tl/MI Hall bar devices with bottom gate electrode.
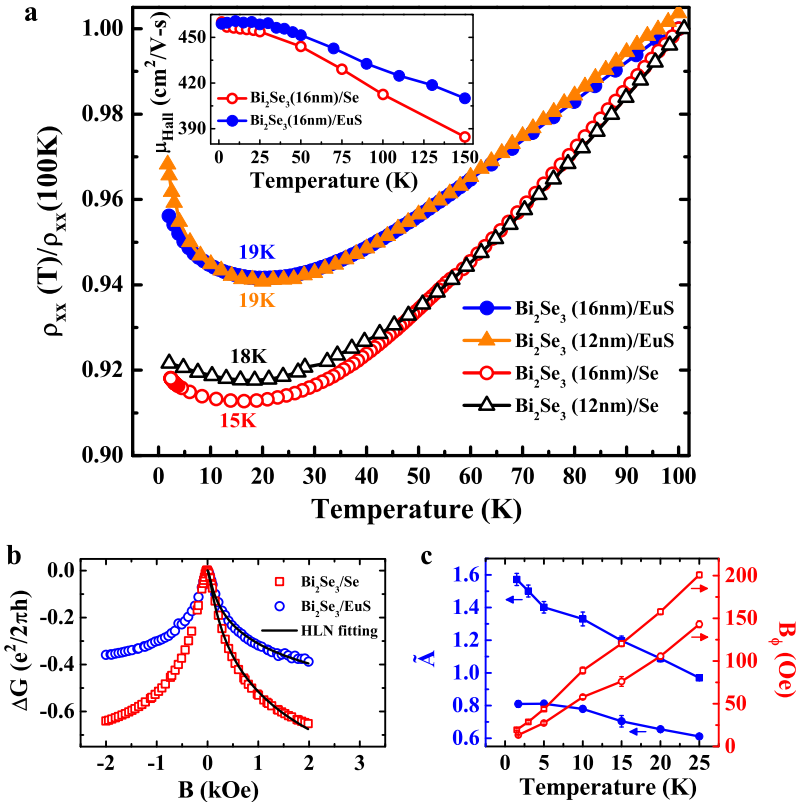

Fig. 2 MIT and WAL response. a Thickness dependence of normalized longitudinal resistivity $\rho_{\mathrm{xx}}$ vs. $T$ for Se-capped (hollow symbols) and EuS-capped device (solid symbols) with $12 \mathrm{~nm}$ (triangles) and $16 \mathrm{~nm}$ (circles) thickness of $\mathrm{Bi}_{2} \mathrm{Se}_{3}$. Corresponding temperature value of the resistivity upturn is also indicated. Inset shows the temperature dependence of $\mu_{\text {Hall }}$ of Se- and EuS-capped 16-nm-thick $\mathrm{Bi}_{2} \mathrm{Se}_{3}$ devices. b Change in magnetoconductance, $\Delta G$ vs. $B$ of $\mathrm{Bi}_{2} \mathrm{Se}_{3} / \mathrm{Se}$ (hollow square) and $\mathrm{Bi}_{2} \mathrm{Se}_{3} / \mathrm{EuS}$ (hollow circle) devices fitted with HLN formalism (solid line). c Temperature dependence of HLN fitting parameters, $\tilde{A}$ (solid) and $B_{\phi}$ (hollow) for $\mathrm{Bi}_{2} \mathrm{Se}_{3} / \mathrm{Se}$ (square) and $\mathrm{Bi}_{2} \mathrm{Se}_{3} /$ EuS (circle) devices.

including the study with thinner films $(<8 \mathrm{~nm})$, report anomalous Hall effect with no evidence of WAL or WL signal after EuS growth. Raman study of these interfaces also reveal strong charge transfer $^{26}$ that may suggest interface hybridization to weaken and destroy the topological signature of SSs. This may not be a surprise since lattice-matched interfaces can sometimes be detrimental for proximity engineering, as also observed in the study of proximity-induced superconductivity ${ }^{27}$.

\section{RESULTS AND DISCUSSION}

Sample preparation

In this article, we adopt a different approach to reduce the bulk conductivity by growing out-of-plane textured $\mathrm{Bi}_{2} \mathrm{Se}_{3}$ films on amorphous $\mathrm{SiO}_{2}\left(\mathrm{a}-\mathrm{SiO}_{2}\right)$ substrate having a hexagonal surface atomic structure that is found essential for promoting the out-ofplane magnetization of EuS ${ }^{18}$. We achieve such a film order by depositing the films at $250^{\circ} \mathrm{C}$ in our molecular beam epitaxy system with a base pressure of $5 \times 10^{-10}$ mbar using a coevaporation technique. After deposition, films are cooled gradually under Se environment and a capping layer is grown at room temperature. X-ray diffraction (XRD) measurements in Fig. 1a reveal (003) family of peaks suggesting a highly $c$-axis oriented layered growth of $\mathrm{Bi}_{2} \mathrm{Se}_{3}$ films that is found to be structurally as good as our epitaxially grown films on c-cut sapphire substrate. Cross-sectional transmission electron microscope (TEM) images (Fig. 1c) also reveal a layered growth supporting the hexagonal structure of $0.95 \mathrm{~nm}$ thickness of the QL with no interfacial strain compared to the films grown on sapphire. Our reflective highenergy electron diffraction study suggested in-plane non-textured growth of the films. Similar growth findings were also noted in the work of Bansal et al. ${ }^{28}$. The structural phase of the $\mathrm{Bi}_{2} \mathrm{Se}_{3}$ films was confirmed using Raman spectroscopy (see Supplementary Fig. 1 and Supplementary Note 1). For transport measurements, thicker $\mathrm{Bi}_{2} \mathrm{Se}_{3}$ films in $10-16 \mathrm{~nm}$ range were grown and, subsequently, a $3 \mathrm{~nm}$ film of EuS was deposited in a sulfur-rich environment at room temperature, without breaking vacuum, followed by a 2-nmcapping layer of $\mathrm{AlO}_{x}(x \sim 1.3-1.5)$. Our XRD analysis showed no EuS peaks, suggesting its amorphous growth.

\section{Metal-to-insulator transition (MIT) and weak anti-localization} response

Hall bar structures with length, $L$ and width, $W$ were patterned by mechanical etching or by in-situ shadow mask (Fig. 1d) as optical lithography techniques degraded the device mobility (see Fig. S3). Hall mobility $\left(\mu_{\text {Hall }}\right)$ of our $16 \mathrm{~nm} \mathrm{Bi}_{2} \mathrm{Se}_{3}$ films was found to be $\sim 450 \mathrm{~cm}^{2} / \mathrm{Vs}$ (inset of Fig. 2a). Figure 2a shows the temperature dependence of normalized longitudinal resistivity $\left(\rho_{\mathrm{xx}}\right)$ for 12 and $16 \mathrm{~nm} \mathrm{Bi} \mathrm{Se}_{3}$ films, grown under the same conditions, with two different capping layers, viz., $2 \mathrm{~nm}$ Se and $3 \mathrm{~nm}$ EuS. Both devices show a predominant metallic signature during cool down with the decrease in $\rho_{\mathrm{xx}}$ for the EuS-capped devices being relatively gradual than the Se-capped devices, a trend observed in different samples with different $W(200 \mu \mathrm{m}$ to $2 \mathrm{~mm})$. At lower temperatures, a weak upward trend in $\rho_{\mathrm{xx}}$ is observed with a stronger 
response in the EuS-capped devices. In the case of Se-capped films, the upturn in $\rho_{\mathrm{xx}}$ occur at $\sim 18 \mathrm{~K}$ for $12 \mathrm{~nm}$ films and decrease to $\sim 15 \mathrm{~K}$ for $16 \mathrm{~nm}$ films. While, in the case of EuScapped films, a stronger upturn in $\rho_{\mathrm{xx}}$ occurs at a temperature of $19 \mathrm{~K}$, that remains unchanged in both the 12 and $16 \mathrm{~nm} \mathrm{Bi} 2 \mathrm{Se}_{3} /$ EuS devices. In general, a weak upturn in film resistivity arises due to localization effects ${ }^{29,30}$ in the $2 \mathrm{D}$ limit originating from the film disorder. However, observation of a stronger upturn in EuScapped devices at a higher temperature (near $T_{c}$ of EuS) than the reference films, while showing no dependence on the $\mathrm{Bi}_{2} \mathrm{Se}_{3}$ film thickness, suggests an additional interface mechanism at play in our EuS-capped devices. Furthermore, our observation of similar $\mu_{\text {Hall }}$ in the EuS-capped and Se-capped devices of Fig. 2a, also strengthen our above claim.

To analyze the influence of the above interface effect on the WAL response of the SSs, magnetoconductance $(G(B), B$ is the magnetic field) study in the low-field range $(B<0.2 \mathrm{~T}$, applied perpendicular to the film) was performed at low temperatures $(<30 \mathrm{~K})$. In Fig. 2 b, a strong upward cusp in $\Delta G(B)=G(B)-G(0)$ is measured at zero field in both the EuS-capped and Se-capped devices with $W / L \sim 1$, providing a clear signature of the presence of 2D WAL channel in our films. The above magnetoconductance plots are fitted with the Hikami-Larkin-Nagaoka (HLN) formalism $^{31}$, given by $\Delta G(B)=\tilde{A} e^{2} / 2 \pi h\left[\ln \left(B_{\phi} / B\right)-\psi\left(1 / 2+B_{\phi} / B\right)\right]$, where $\psi(x)$ is the digamma function, $e$ is electronic charge, and $h$ is the Planck's constant. The temperature dependency of the fitting parameters, dephasing field $\left(B_{\phi}\right)$ and prefactor $\tilde{A}$, that denote the effective number of $2 D$ conducting channels contributing to WAL is plotted in Fig. 2c. In this low-field range, the contribution of bulk states to the WAL response, determined by performing in-plane magnetoconductance measurements ${ }^{32}$, was found to be negligible without significantly affecting the parameters extracted from the HLN fitting. In TI, we have two 2D WAL channels corresponding to the bottom and top SSs. Proximity of one of the surface (top) with MI, which theoretically opens an exchange gap, can considerably weaken its WAL response. For the case of Se-capped devices, we find $\tilde{A} \sim 1.6$, which indicates good decoupling between the top and bottom $2 \mathrm{D}$ WAL channels. However, in the EuS-capped devices, we notice a drop in WAL channel by $\sim 0.8$ at $1.5 \mathrm{~K}$ suggesting additional localization response due to EuS capping (see Fig. 2c).

Our above findings of localization at the top $\mathrm{Bi}_{2} \mathrm{Se}_{3} / E u S$ interface can arise due to different possible reasons. To rule out its origin to any trivial response from the EuS layer, temperature dependence of resistivity of a trivial metal, $\mathrm{Pt}(5 \mathrm{~nm})$, was measured that showed no change in behavior due to its proximity with EuS ( $2 \mathrm{~nm}$ ) (see Supplementary Fig. 11). These observations limit our explanation for the origin of the above response to possible electron-electron interactions (EEI) ${ }^{33}$ or MPE-induced EG opening $^{19}$. In the case of EEl, the large g-factor of the SSs can activate a large Zeeman splitting from the exchange field of EuS and contribute to corrections in the conductivity. In the following discussions, we refer to the above localization in EuS-capped films, causing an upturn in film resistivity, to an MIT response of the SSs.

\section{Interface band-bending effects}

In Tls, the position of $E_{F}$ at the surface can greatly depend on the relative concentration of bulk and surface defect density ${ }^{29,34}$. A large mismatch in the surface and bulk $E_{F}$ can lead to charge transfer and bending of bulk bands near the surface, resulting in a depleted (an accumulated) surface region in films with lower (higher) surface defect density ${ }^{35,36}$ (refer to Supplementary Note 4). Such surface band-bending response, creating interfacial electrostatic fields that shift the Dirac cone and position of the $E_{F}$ at the interface, has been observed using angle-resolved photoemission spectroscopy (ARPES) ${ }^{37}$ and gated terahertz cyclotron resonance measurements ${ }^{38}$. In our films, the high bulk carrier
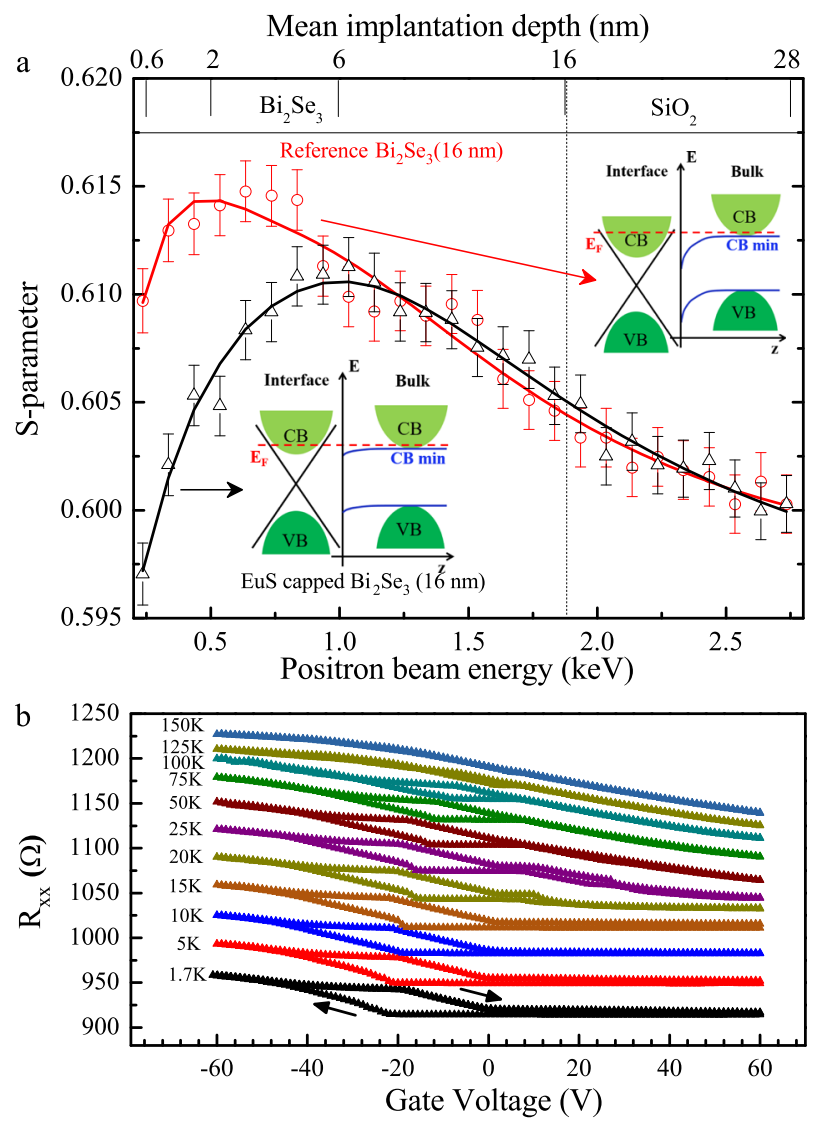

Fig. 3 PAS and interface band-bending effects. a S-parameter as a function of positron beam energy for reference $\mathrm{Bi}_{2} \mathrm{Se}_{3}$ film and EuScapped $\mathrm{Bi}_{2} \mathrm{Se}_{3}$ film. The mean implantation depth probed by the positron beam is shown on the top axis. VEPFIT deduced $S$ vs. $E_{p}$ curves are shown by solid lines. Inset shows schematic of bandbending profile near the surface of $\mathrm{Bi}_{2} \mathrm{Se}_{3}$. The error bar in the figure shows the standard error of the mean. b $R_{x x}$ vs. $V_{g}$ of the $\mathrm{Bi}_{2} \mathrm{Se}_{3}(16 \mathrm{~nm}) / \mathrm{EuS}(3 \mathrm{~nm})$ device measured at different Ts. A second hysteresis response appears in positive $V_{g}$ at and below $25 \mathrm{~K}$ (refer to Supplementary Fig. 6). The plots are shifted vertically for clarity.

density $\left(>10^{19} / \mathrm{cm}^{3}\right)$ is expected to pin the bulk $E_{F}$ in the $C B$. However, understanding surface band-bending effects can help locate the position of $E_{F}$ at the interface and to qualitatively probe the origin of the above interfacial MIT response.

In our above effort, we performed depth-resolved positron annihilation spectroscopy (PAS) measurements at room temperature using variable low-energy positron beam that allowed profiling the vacancy-type defects ${ }^{39,40}$, i.e., primarily the Se vacancies (see "Methods"). From the Doppler-broadened positron annihilation spectrum, $S$-parameter, defined as the ratio of the counts in $511 \pm 1 \mathrm{keV}$ peak region to the total counts in $511 \pm$ $10 \mathrm{keV}$ region, is deduced. The $S$-parameter characterizes the annihilation of positrons with low-momentum valence electrons, which is directly related to the concentration of vacancy defects. In Fig. 3a, we plot the $S$-parameter, extracted from the positron annihilation spectrum, vs. positron beam energy $\left(E_{p}\right) /$ mean implantation depth for the reference $\mathrm{Bi}_{2} \mathrm{Se}_{3}$ and the $\mathrm{Bi}_{2} \mathrm{Se}_{3} / \mathrm{EuS}$ films having a capping layer of $\mathrm{AlO}_{x}$. Here, the effect of variation in the capping layer thickness for the two films has been included in the PAS analysis (see Supplementary Note 7). We observe that for very low positron energies, the $S$-parameter is higher for the reference $\mathrm{Bi}_{2} \mathrm{Se}_{3}$ films than the EuS-capped films. As the energy increases beyond $\sim 1.25 \mathrm{keV}$, corresponding to an implantation depth of $\sim 9 \mathrm{~nm}$, the $S$-parameter for the two samples merge suggesting similar bulk response of the $\mathrm{Bi}_{2} \mathrm{Se}_{3}$ films (see 

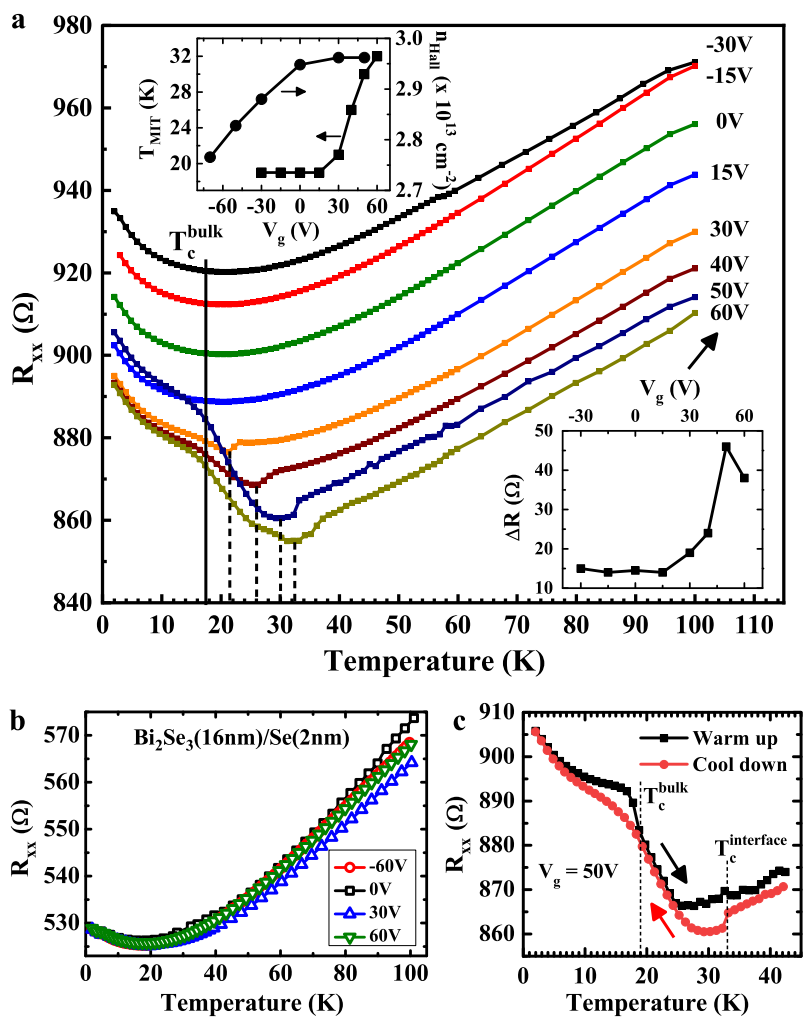

Fig. 4 Gate tunable interface magnetism with enhancement in $\boldsymbol{T}_{\boldsymbol{c}}$. a $R_{\mathrm{xx}}$ vs. $T$ of $\mathrm{Bi}_{2} \mathrm{Se}_{3}(16 \mathrm{~nm}) / \mathrm{EuS}(3 \mathrm{~nm})$ device $(L=1.5 \mathrm{~mm}, W=1 \mathrm{~mm})$ measured at different $V_{g}$ s. $T_{c}^{\text {bulk }}$ denotes the $T_{c}$ of the bulk EuS film, measured at negative $V_{g}$ (depleted carriers at interface). Top left inset shows increase in $T_{\text {MIT }}$ (square; shown by vertical dashed lines in main figure) at positive $V_{g} s$ which is corroborated by the increase in $n_{\text {Hall }}$ (circle). Enhanced interface magnetism, attributed to indirect exchange at positive $V_{g} \mathrm{~s}$, leads to large $\Delta R=R(1.5 \mathrm{~K})-R$ $\left(T_{\mathrm{MIT}}\right)$ peaking at $V_{g}=50 \mathrm{~V}$ (bottom-right inset). b $R_{\mathrm{xx}} \mathrm{vs}$. $T$ of $\mathrm{Bi}_{2} \mathrm{Se}_{3}(16 \mathrm{~nm}) / \mathrm{Se}(2 \mathrm{~nm})$ device at different $V_{g}$ s, c Warm-up and cooldown plot of $R_{\mathrm{xx}} \mathrm{Vs}$. $T$ at $V_{g}=50 \mathrm{~V}$ showing hysteresis response for $T<19 \mathrm{~K}$ and $T>25 \mathrm{~K}$ attributed to different $T_{c}$ of bulk $(19 \mathrm{~K})$ and interface $(33 \mathrm{~K})$ EuS, respectively.

Supplementary Note 7 for detailed analysis). Variable energy positron fit (VEPFIT) software analysis ${ }^{41}$ was carried out by assuming a two-layer fitting, comprising of the $\mathrm{Bi}_{2} \mathrm{Se}_{3}$ film and the underneath $\mathrm{SiO}_{2}$ substrate layer (see Supplementary Note 7). The above analysis to the experimental $S$ vs. $E_{p}$ curve yielded best fits by invoking a mean internal electric field in the $\mathrm{Bi}_{2} \mathrm{Se}_{3}$ film within the depth of $9 \mathrm{~nm}$ from the top TI surface. In the reference $\mathrm{Bi}_{2} \mathrm{Se}_{3}$ samples, a large positive interfacial electric field of $6.1 \times$ $10^{-4} \mathrm{~V} / \mathrm{nm}$ was extracted supporting the formation of a downward band bending (top right inset of Fig. 3a), as also observed in ARPES study of $\mathrm{Bi}_{2} \mathrm{Se}_{3}$ surfaces ${ }^{37}$. On the contrary, in the EuScapped films, an order of magnitude drop in the mean electric field is observed $\left(\sim 0.9 \times 10^{-4} \mathrm{~V} / \mathrm{nm}\right)$. Considering the interfacial electric field to be proportional to the carrier density, we find that the surface carrier density at the $\mathrm{Bi}_{2} \mathrm{Se}_{3} / E$ uS interface (compared to bulk value of $\sim 4 \times 10^{13} / \mathrm{cm}^{2}$ ) can fall by nearly an order of magnitude to $\sim 10^{12} / \mathrm{cm}^{2}$. These interpretations are in agreement with density functional theory (DFT) studies that reveal Eu-Se bond formation at the $\mathrm{Bi}_{2} \mathrm{Se}_{3} / \mathrm{EuS}$ interface to be more favorable ${ }^{23}$ leading to a lower surface defect density. Such a condition support a nearly flat-band condition moving the interface $E_{F}$ closer to the band edge (bottom inset of Fig. 3a, also see Supplementary Note 4).

In Fig. 3b, we show temperature dependence of longitudinal resistance $\left(R_{x x}\right)$ vs. back-gate voltage $\left(V_{g}\right)$ of the $\mathrm{Bi}_{2} \mathrm{Se}_{3} / \mathrm{EuS}$ devices. Interestingly, a hysteresis response in $R_{\mathrm{xx}}$ is observed at all temperatures below $125 \mathrm{~K}$, with similar response observed in four different sets of samples and with different film thickness (see Supplementary Note 6 and Supplementary Fig. 6). In comparison, the Se-capped devices showed no such behavior (see Supplementary Note 5 and Supplementary Fig. 5). Presence of such hysteresis response observed only in $\mathrm{Bi}_{2} \mathrm{Se}_{3}$ /EuS devices at low temperatures further supports our PAS analysis that the interface $E_{F}$ lies near the band edge and drops into the band gap with cool down due to freezing of charge carriers. With the position of interface $E_{F}$ in the band gap, $V_{g}$ modulation leads to pinning/depinning effects at the band edge, responsible for the observed hysteresis in Fig. 3b. These findings may therefore suggest the origin of our interfacial MIT response to the localization affects around the Dirac point of the top SSs.

\section{Effect of gate voltage on interface magnetism}

The MIT response in our $\mathrm{Bi}_{2} \mathrm{Se}_{3} /$ EuS devices was further investigated in Fig. 4 a by measuring $R_{\mathrm{xx}}$ vs. $T$ at different $V_{g} s$. Here, at negative $V_{g}$, an insulating transition was observed at temperature, $T_{\mathrm{MIT}} \sim 19 \mathrm{~K}$, which was enhanced to $\sim 32 \mathrm{~K}$ at positive $V_{g}$ with a corresponding increase in the carrier density (top left inset of Fig. 4a). In comparison, the Se-capped devices showed no such response (Fig. 4b). Such an increase in $T_{\text {MIT }}$ at positive $V_{g}$, which also makes the device conducting (seen by the downward shift of plots), at first seems counter-intuitive. However, this observation may be suggestive of conduction-electron-mediated ferromagnetic exchange between the Eu atoms strengthening the interface magnetism, with an enhancement in $T_{c}$ of the EuS "interface layer". This enhanced interface magnetism in our devices is resulting in a larger increase in resistance below $T_{\text {MIT, }}$, peaking at $V_{g} \sim 50 \mathrm{~V}$ (bottom-right inset of Fig. 4a). Comparing with the warm-up data of $R_{\mathrm{xx}}$ vs. $T$ (Fig. 4c), we observe a clear hysteresis in the low- and high-field range that supports the existence of different magnetic transition temperature of bulk $\left(T_{c}^{\text {bulk }}\right)$ and interface ( $T_{c}^{\text {interface}}$ ) EuS layer. This feature is also revealed in resistance plots $\left(V_{g}>15 \mathrm{~V}\right)$ in Fig. 4a with a point of inflection at $19 \mathrm{~K}$, below which, the magnetic transition of EuS layers away from the interface causes the resistance to increases further. Similar increase in $T_{c}$ of EuS, however, due to bulk electron doping has been previously reported ${ }^{42,43}$. It is interesting to note that at large negative $V_{g}$ (depleted interface), we observe a higher value of $T_{\text {MIT }} \sim 19 \mathrm{~K}$, i.e., $2 \mathrm{~K}$ above the bulk $T_{c}$ of EuS $(17 \mathrm{~K})$ which may indicate the role of high spin-orbit strength of the $\mathrm{Bi}_{2} \mathrm{Se}_{3}$ $\mathrm{SSs}^{20}$.

In EuS, magnetism arises from the overlap of the Eu $4 f$ orbitals with the valence band below the $E_{F}$ that leads to a weaker exchange coupling ${ }^{44}$. DFT studies by Kim et al. ${ }^{20}$ show that the SSs that weakly interconnect the conduction and valance bands can mediate exchange coupling between $\mathrm{Eu}$ atoms through the Ruderman-Kittel-Kasuya-Yosida (RKKY) type indirect interactions ${ }^{45-47}$, with the exchange constant being sensitive to the position of $E_{F}$ near the Dirac point. The observed gate-controlled tuning of $T_{\text {MIT }}$ in our devices provides evidence for such an indirect exchange mechanism existing at the $\mathrm{Bi}_{2} \mathrm{Se}_{3} / \mathrm{EuS}$ interface. Also, cooling the device in perpendicular magnetic fields $\left(H_{\mathrm{FC}}\right)$, at $V_{g}=50 \mathrm{~V}$ showed a surprising decrease in $T_{\mathrm{MIT}}$ (or $T_{c}^{\text {interface }}$ ), with the increasing strength of $H_{\mathrm{FC}}$ (see Fig. 5). Such a response may arise because, here, the external magnetic field interacts not only with the localized $4 f$ spins of Eu by direct interaction, but also through the conduction electrons at the interface. Such a theoretical framework of RKKY interactions that include the sign and value of the interaction potential between the localized spins and the polarized conduction electrons in external magnetic field has not been extensively explored ${ }^{48}$. In addition, EEls in presence of Zeeman field can also greatly affect the above mentioned indirect interactions causing a reduction in $T_{c}^{\text {interface } 49}$. 


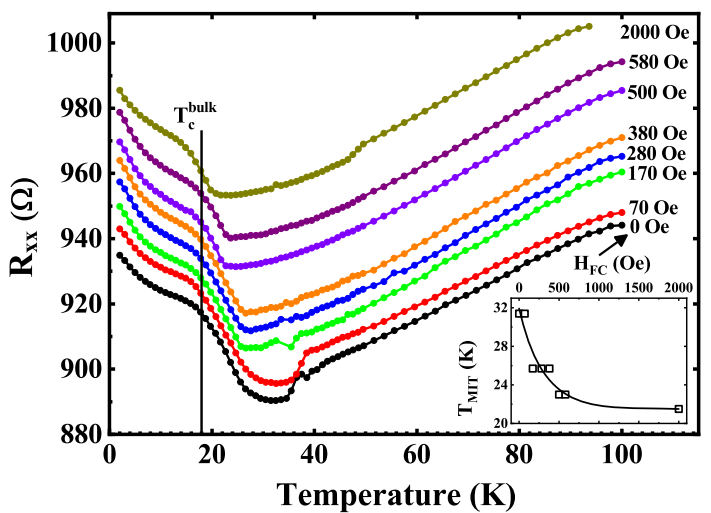

Fig. 5 Effect of field cooling on the MIT response. $R_{\mathrm{xx}}$ vs. $T$ of the $\mathrm{Bi}_{2} \mathrm{Se}_{3}(16 \mathrm{~nm}) / \mathrm{EuS}(3 \mathrm{~nm})$ device measured at $V_{g}=50 \mathrm{~V}$ with varying perpendicular $H_{\mathrm{FC}} \mathrm{S}$. The plots are shifted vertically for clarity. Bottom-right inset shows lowering of $T_{\mathrm{MIT}}$ with $H_{\mathrm{FC}}$ attributed to the weakening of the indirect exchange at the interface.

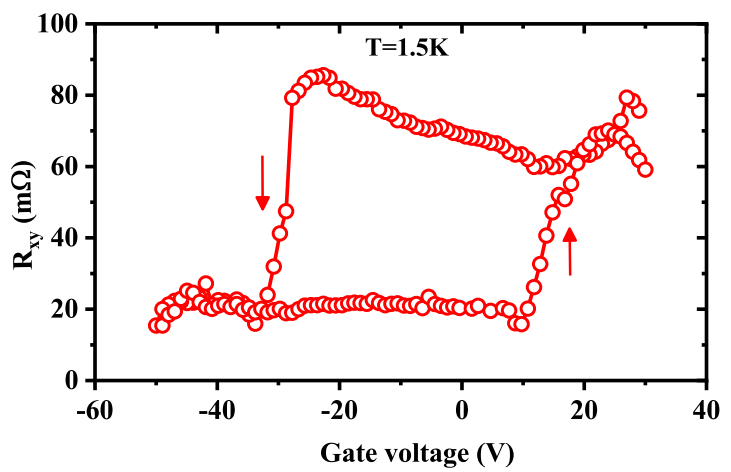

Fig. 6 Gate voltage dependence of Hall resistance. $R_{\mathrm{xy}}$ vs. $V_{g}$ for $\mathrm{Bi}_{2} \mathrm{Se}_{3}(16 \mathrm{~nm}) / \mathrm{EuS}(3 \mathrm{~nm})$ device at $1.5 \mathrm{~K}$.

Gate voltage dependence of Hall signal

Apart from the enhancement in $T_{\mathrm{MIT}}$ for $V_{g}>15 \mathrm{~V}$ in Fig. $4 \mathrm{a}$, we also observe a prominent and reproducible drop in device resistance preceding MIT. This sudden drop in resistance shows a maximum of $\sim 10 \Omega$ at $V_{g}=50 \mathrm{~V}$. Also, when cooled in higher magnetic fields, the resistance drop preceding $T_{\mathrm{MIT}}$ starts to vanish (Fig. 5). Although the increase in $R_{\mathrm{xx}}$ due to magnetic proximity is in agreement with the localization effects observed from our WAL studies, the concomitant drop in $R_{\mathrm{xx}}$ at the onset of interface magnetism is not well explained based on any spin disorder or EEI models supporting WL mechanisms ${ }^{16,33,50}$. In Fig. 6, we plot Hall resistance $\left(R_{\mathrm{xy}}\right)$ vs. $V_{g}$ of the device, measured at $1.5 \mathrm{~K}$, that showed a hysteresis response with the value rising above $V_{g} \sim 15 \mathrm{~V}$ in the positive sweep cycle reaching $\sim 70 \mathrm{~m} \Omega$ and dropping down at $-27 \mathrm{~V}$ in the negative sweep cycle of $V_{g}$. Comparing with the data of Fig. 4a where the resistance drop in $R_{\mathrm{xx}}$ preceding $T_{\mathrm{MIT}}$ is observed only for $V_{g}>15 \mathrm{~V}$, we notice an increase in $R_{x y}$ in the same $V_{g}$ window (during the positive $V_{g}$ sweep cycle) in Fig. 6 that might suggest a common phenomenon responsible for the above two responses. We have analyzed the possibility to associate the above origin to a weak signature of $\mathrm{H}-\mathrm{IQHE}$ driven by the movement of $E_{F}$ in the EG (see Supplementary Note 8). However, the weak experimental signal, possibly due to the large bulk conductivity, canted magnetism of EuS and finite temperature makes this analysis inconclusive and open for future exploration.

In conclusion, we report favorable growth conditions of EuSproximitized $\mathrm{Bi}_{2} \mathrm{Se}_{3}$ films on a-SiO${ }_{2}$ substrate providing evidence of lower vacancy-type surface defect density that support the movement of the interface $E_{F}$ toward the Dirac point. Also, we observe localization response in the proximitized SSs below the $T_{c}$ of EuS with a possible topological origin. Most importantly, we demonstrate the possibility to modulate interface magnetism using $V_{g}$, driven by an indirect RKKY interaction at the $\mathrm{Bi}_{2} \mathrm{Se}_{3} /$ EuS interface. These findings offer us the possibility to design a more robust surface quantum Hall system in our quest to develop switchable topological electronic devices.

\section{METHODS}

Growth, device fabrication, and transport measurement

Film growth was performed in a custom designed molecular beam epitaxy system with a base pressure of $5 \times 10^{-10} \mathrm{mbar}$ with all crucial interfaces prepared without breaking vacuum. Hall bar devices were mechanically patterned under a microscope and contacts were made using wire bonding on a 44 leadless chip carrier (refer to Supplementary Note 3). In addition, Hall bar geometry devices were also fabricated using in-situ shadow mask technique. Low-temperature magneto-transport measurements were performed in a variable temperature insert cryostat with a base temperature of $1.5 \mathrm{~K}$.

\section{Positron annihilation spectroscopy}

Depth-resolved Doppler broadening measurements were carried out on the samples using the variable low-energy positron beam. Using this approach, vacancy-type defects were studied in our films starting from the surface up to a depth of a few hundreds of $\mathrm{nm}$ by implanting positron beam of varying energy ranging from $235 \mathrm{eV}$ to $2.735 \mathrm{keV}$ in steps of $0.1 \mathrm{keV}$. The mean implantation depth follows an empirical relation: $z(\mathrm{~nm})=\left(40 \times E_{p}^{1.6}\right) / \rho$ where $E_{p}$ is the positron beam energy in $\operatorname{keV}$ and $\rho$ is the density of the sample in $\mathrm{g} / \mathrm{cm}^{3}$. For the PAS study, Doppler broadened $511 \mathrm{keV}$ annihilation energy spectrum was obtained for each positron beam energy using HPGe detector having an energy resolution of $1.5 \mathrm{keV}$ for $662 \mathrm{keV} \gamma$-ray of ${ }^{137} \mathrm{Cs}$. From each annihilation spectrum, the $S$-parameter was deduced.

\section{DATA AVAILABILITY}

The data that support the findings of this study are available from the corresponding author upon reasonable request.

Received: 27 January 2020; Accepted: 28 July 2020; Published online: 09 September 2020

\section{REFERENCES}

1. Hasan, M. Z. \& Kane, C. L. Colloquium: topological insulators. Rev. Mod. Phys. 82 3045-3067 (2010).

2. Qi, X. L. \& Zhang, S. C. Topological insulators and superconductors. Rev. Mod. Phys. 83, 1057-1110 (2011).

3. Chen, Y. L. et al. Massive Dirac fermion on the surface of a magnetically doped topological insulator. Science 329, 659-662 (2010).

4. Yu, R. et al. Quantized anomalous Hall effect in magnetic topological insulators. Science 329, 61-64 (2010).

5. Chang, C.-Z. et al. Experimental observation of the quantum anomalous Hall effect in a magnetic topological insulator. Science 340, 167-170 (2013).

6. Gusynin, V. P. \& Sharapov, S. G. Unconventional integer quantum Hall effect in graphene. Phys. Rev. Lett. 95, 146801 (2005).

7. Qi, X.-L., Hughes, T. L. \& Zhang, S.-C. Topological field theory of time-reversal invariant insulators. Phys. Rev. B 78, 195424 (2008).

8. Essin, A. M., Moore, J. E. \& Vanderbilt, D. Magnetoelectric polarizability and axion electrodynamics in crystalline insulators. Phys. Rev. Lett. 102, 146805 (2009).

9. Qi, X.-L., Li, R., Zang, J. \& Zhang, S.-C. Inducing a magnetic monopole with topological surface states. Science 323, 1184-1187 (2009).

10. Mogi, M. et al. Tailoring tricolor structure of magnetic topological insulator for robust axion insulator. Sci. Adv. 3, eaao1669 (2017).

11. Hor, Y. S. et al. Development of ferromagnetism in the doped topological insulator $\mathrm{Bi}_{2-x} \mathrm{Mn}_{x} \mathrm{Te}_{3}$. Phys. Rev. B 81, 195203 (2010).

12. Hou, Y., Kim, J. \& Wu, R. Magnetizing topological surface states of $\mathrm{Bi}_{2} \mathrm{Se}_{3}$ with a $\mathrm{Crl}_{3}$ monolayer. Sci. Adv. 5, eaaw1874 (2019).

13. Men'shov, V. N., Tugushev, V., Eremeev, S., Echenique, P. \& Chulkov, E. Magnetic proximity effect in the three-dimensional topological insulator/ferromagnetic insulator heterostructure. Phys. Rev. B 88, 224401 (2013). 
14. Luo, W. \& Qi, X.-L. Massive Dirac surface states in topological insulator/magnetic insulator heterostructures. Phys. Rev. B 87, 085431 (2013).

15. Eremeev, S. V. et al. Magnetic proximity effect at the three-dimensional topological insulator/magnetic insulator interface. Phys. Rev. B 88, 144430 (2013).

16. Eremeev, S. V., Men'shov, V. N., Tugushev, V. V. \& Chulkov, E. V. Interface induced states at the boundary between a $3 \mathrm{~d}$ topological insulator $\mathrm{Bi}_{2} \mathrm{Se}_{3}$ and a ferromagnetic insulator EuS. J. Magn. Magn. Mater. 383, 30-33 (2015).

17. Wei, P. et al. Exchange-coupling-induced symmetry breaking in topological insulators. Phys. Rev. Lett. 110, 186807 (2013).

18. Katmis, F. et al. A high-temperature ferromagnetic topological insulating phase by proximity coupling. Nature 533, 513-516 (2016).

19. Yang, Q. I. et al. Emerging weak localization effects on a topological insulator-insulating ferromagnet $\mathrm{Bi}_{2} \mathrm{Se}_{3}$-EuS interface. Phys. Rev. B 88, 081407 (2013).

20. Kim, J., Kim, K.-W., Wang, H., Sinova, J. \& Wu, R. Understanding the giant enhancement of exchange interaction in $\mathrm{Bi}_{2} \mathrm{Se}_{3}$-EuS heterostructures. Phys. Rev. Lett. 119, 027201 (2017).

21. Lang, M. et al. Proximity induced high-temperature magnetic order in topological insulator-ferrimagnetic insulator heterostructure. Nano Lett. 14, 3459-3465 (2014).

22. Liu, W. et al. Enhancing magnetic ordering in cr-doped $\mathrm{Bi}_{2} \mathrm{Se}_{3}$ using high- $T_{c}$ ferrimagnetic insulator. Nano Lett. 15, 764-769 (2015).

23. Lee, A. T., Han, M. J. \& Park, K. Magnetic proximity effect and spin-orbital texture at the $\mathrm{Bi}_{2} \mathrm{Se}_{3} /$ EuS interface. Phys. Rev. B 90, 155103 (2014).

24. Krieger, J. A. et al. Do topology and ferromagnetism cooperate at the $\mathrm{EuS} / \mathrm{Bi}_{2} \mathrm{Se}_{3}$ interface? Phys. Rev. B 99, 064423 (2019).

25. Yang, $X$. et al. Light control of surface-bulk coupling by terahertz vibrational coherence in a topological insulator. npj Quantum Mater. 5, 1-7 (2020).

26. Osterhoudt, G. B. et al. Charge transfer in $\mathrm{EuS} / \mathrm{Bi}_{2} \mathrm{Se}_{3}$ heterostructures as indicated by the absence of raman scattering. Phys. Rev. B 98, 014308 (2018).

27. Reeg, C., Loss, D. \& Klinovaja, J. Metallization of a rashba wire by a superconducting layer in the strong-proximity regime. Phys. Rev. B 97, 165425 (2018).

28. Bansal, N. et al. Robust topological surface states of $\mathrm{Bi}_{2} \mathrm{Se}_{3}$ thin films on amorphous $\mathrm{SiO}_{2} / \mathrm{Si}$ substrate and a large ambipolar gating effect. Appl. Phys. Lett. 104, 241606 (2014).

29. Brahlek, M., Koirala, N., Bansal, N. \& Oh, S. Transport properties of topological insulators: band bending, bulk metal-to-insulator transition, and weak antilocalization. Solid State Commun. 215, 54-62 (2015).

30. Brahlek, M., Koirala, N., Salehi, M., Bansal, N. \& Oh, S. Emergence of decoupled surface transport channels in bulk insulating $\mathrm{Bi}_{2} \mathrm{Se}_{3}$ thin films. Phys. Rev. Lett. 113, 026801 (2014).

31. Hikami, S., Larkin, A. I. \& Nagaoka, Y. Spin-orbit interaction and magnetoresistance in the two dimensional random system. Prog. Theor. Phys. 63, 707-710 (1980).

32. He, H.-T. et al. Impurity effect on weak antilocalization in the topological insulator $\mathrm{Bi}_{2} \mathrm{Te}_{3}$. Phys. Rev. Lett. 106, 166805 (2011).

33. Wang, J. et al. Evidence for electron-electron interaction in topological insulator thin films. Phys. Rev. B 83, 245438 (2011).

34. Veyrat, $\mathrm{L}$. et al. Band bending inversion in $\mathrm{Bi}_{2} \mathrm{Se}_{3}$ nanostructures. Nano Lett. 15, 7503-7507 (2015)

35. Benia, H. M., Lin, C., Kern, K. \& Ast, C. R. Reactive chemical doping of the $\mathrm{Bi}_{2} \mathrm{Se}_{3}$ topological insulator. Phys. Rev. Lett. 107, 177602 (2011).

36. Rakyta, P., Ujfalussy, B. \& Szunyogh, L. Band bending at the surface of $\mathrm{Bi}_{2} \mathrm{Se}_{3}$ studied from first principles. N. J. Phys. 17, 123011 (2015).

37. Hsieh, D. et al. A tunable topological insulator in the spin helical Dirac transport regime. Nature 460, 1101-1105 (2009).

38. Jenkins, G. S. et al. Dirac cone shift of a passivated topological $\mathrm{Bi}_{2} \mathrm{Se}_{3}$ interface state. Phys. Rev. B 87, 155126 (2013).

39. Amarendra, G., Viswanathan, B., Rao, G. V., Parimala, J. \& Purniah, B. Variable low energy positron beams for depth resolved defect spectroscopy in thin film structures. Curr. Sci. 73, 409-417 (1997).

40. Schultz, P. \& Lynn, K. Interaction of positron beams with surfaces, thin films, and interfaces. Rev. Mod. Phys. 60, 701-779 (1988).

41. Veen, A. V., Schut, H., de Vries, J., Hakvoort, R. A. \& ljpma, M. R. Analysis of positron profiling data by means of "VEPFIT". AIP Conf. Proc. 218, 171-198 (1991).

42. Holtzberg, F., McGuire, T. R., Methfessel, S. \& Suits, J. C. Effect of electron concentration on magnetic exchange interactions in rare earth chalcogenides. Phys. Rev. Lett. 13, 18 (1964).

43. Selinsky, R. S., Han, J. H., Pérez, E. A. M., Guzei, I. A. \& Jin, S. Synthesis and magnetic properties of Gd doped EuS nanocrystals with enhanced Curie temperatures. J. Am. Chem. Soc. 132, 15997-16005 (2010)
44. Coey, J. M. D., Viret, M. \& Molnar, S. V. Mixed-valence manganites. Adv. Phys. 48, 167-293 (1999).

45. Ruderman, M. A. \& Kittel, C. Indirect exchange coupling of nuclear magnetic moments by conduction electrons. Phys. Rev. C 96, 99 (1954).

46. Kasuya, T. A. A theory of metallic ferro-and antiferromagnetism on zener's model. progress of theoretical physics. Prog. Theory Phys. 16, 45-57 (1956).

47. Yosida, K. Magnetic properties of Cu-Mn alloys. Phys. Rev. 106, 893 (1957).

48. Balcerzak, T. The RKKY interaction in the presence of an external magnetic field. Phys. Status Solid C 3, 212-215 (2006).

49. Ziener, C. H., Glutsch, S. \& Bechstedt, F. RKKY interaction in semiconductors: effects of magnetic field and screening. Phy. Rev. B 70, 075205 (2004).

50. Dzero, M., Xia, J., Galitski, V. \& Coleman, P. Topological kondo insulators. Annu. Rev. Condens. Matter Phys. 7, 249-280 (2016).

\section{ACKNOWLEDGEMENTS}

We would like to thank Dr. G. Amerandra and Dr. R. Rajaraman from IGCAR for fruitful discussions on PAS measurement and analysis and Dr. Mukherjee and Dr. Suri for discussions. S.M. acknowledges SERB NPDF fellowship. We thank T.N. Narayanan for giving access to Raman facility. We thank IIT Hyderabad for providing access to their XRD instrumentation facility and IIT Chennai for SQUID facility. All authors acknowledge extra-mural funding from TIFR-Hyderabad and Department of Atomic Energy (DAE), SERB Sanction Nos. ECR/2015/000199 and CRG/2019/003810.

\section{AUTHOR CONTRIBUTIONS}

S.M. and S.S. fabricated the devices and performed the measurements. A.B., S.S., and R.P. optimized and characterized the TI film growth. S.S. and S.C. setup the measurement facility. S.A. performed PAS measurements. B.S. performed TEM study and the analysis. S.S. and K.V.R. analyzed the data. K.V.R. conceived the idea. S.S. and K.V.R. wrote the paper. All authors contributed to the discussion.

\section{COMPETING INTERESTS}

The authors declare no competing interests.

\section{ADDITIONAL INFORMATION}

Supplementary information is available for this paper at https://doi.org/10.1038/ s41535-020-00267-5.

Correspondence and requests for materials should be addressed to K.V.R.

Reprints and permission information is available at http://www.nature.com/ reprints

Publisher's note Springer Nature remains neutral with regard to jurisdictional claims in published maps and institutional affiliations.

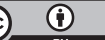

Open Access This article is licensed under a Creative Commons Attribution 4.0 International License, which permits use, sharing, adaptation, distribution and reproduction in any medium or format, as long as you give appropriate credit to the original author(s) and the source, provide a link to the Creative Commons license, and indicate if changes were made. The images or other third party material in this article are included in the article's Creative Commons license, unless indicated otherwise in a credit line to the material. If material is not included in the article's Creative Commons license and your intended use is not permitted by statutory regulation or exceeds the permitted use, you will need to obtain permission directly from the copyright holder. To view a copy of this license, visit http://creativecommons. org/licenses/by/4.0/.

(c) The Author(s) 2020 